University of Wollongong

Research Online

Australian Institute for Innovative Materials -

Papers

Australian Institute for Innovative Materials

$1-1-2017$

Intrinsic and spatially nonuniform ferromagnetism in Co-doped ZnO films

L T. Tseng

University of New South Wales

A Suter

Paul Scherrer Institute

Y R. Wang

University of New South Wales

Feixiang Xiang

University of Wollongong, fx963@uowmail.edu.au

P Bian

University of Sydney

See next page for additional authors

Follow this and additional works at: https://ro.uow.edu.au/aiimpapers

Part of the Engineering Commons, and the Physical Sciences and Mathematics Commons

Research Online is the open access institutional repository for the University of Wollongong. For further information contact the UOW Library: research-pubs@uow.edu.au 


\title{
Intrinsic and spatially nonuniform ferromagnetism in Co-doped ZnO films
}

\author{
Abstract \\ Co doped ZnO films have been deposited by a laser-molecular beam epitaxy system. X-ray diffraction and \\ UV spectra analysis show that Co effectively substitutes the $\mathrm{Zn}$ site. Transmission electron microscopy \\ (TEM) and secondary ion mass spectroscopy analysis indicate that there are no clusters. Co dopants are \\ uniformly distributed in $\mathrm{ZnO}$ film. Ferromagnetic ordering is observed in all samples deposited under an \\ oxygen partial pressure, $\mathrm{PO} 2=10-3,10-5$, and $10-7$ torr, respectively. However, the magnetization of \\ $\mathrm{PO} 2=10-3$ and $10-5$ is very small at room temperature. At low temperature, the ferromagnetic ordering is \\ enhanced. Muon spin relaxation ( $\mu \mathrm{SR}$ ) measurements confirm the ferromagnetism in all samples, and the \\ results are consistent with magnetization measurements. From $\mu$ SR and TEM analysis, the film deposited \\ under P02=10-7 torr shows intrinsic ferromagnetism. However, the volume fraction of the \\ ferromagnetism phase is approximately $70 \%$, suggesting that the ferromagnetism is not carrier mediated. \\ Resistivity versus temperature measurements indicate Efros variable range hopping dominates the \\ conductivity. From the above results, we can confirm that a bound magnetic polaron is the origin of the \\ ferromagnetism. \\ Disciplines \\ Engineering | Physical Sciences and Mathematics

\section{Publication Details} \\ Tseng, L. T., Suter, A., Wang, Y. R., Xiang, F., Bian, P., Ding, X., Tseng, A., Hu, H. L., Fan, H. M., Zheng, R. K., \\ Wang, X. L., Salman, Z., Prokscha, T., Suzuki, K., Liu, R., Li, S., Morenzoni, E. \& Yi, J. B. (2017). Intrinsic and \\ spatially nonuniform ferromagnetism in Co-doped ZnO films. Physical Review B: Condensed Matter and \\ Materials Physics, 96 (10), 104423-1-104423-10.
}

\section{Authors}

L T. Tseng, A Suter, Y R. Wang, Feixiang Xiang, P Bian, X Ding, A Tseng, H L. Hu, Huiqing Fan, Rongkun Zheng, Xiaolin Wang, Z Salman, T Prokscha, Katsuaki Suzuki, R Liu, Sean Li, E Morenzoni, and Jiabao Yi 


\title{
Intrinsic and spatially nonuniform ferromagnetism in Co-doped $\mathrm{ZnO}$ films
}

\author{
L. T. Tseng, ${ }^{1}$ A. Suter, ${ }^{2}$ Y. R. Wang, ${ }^{1}$ F. X. Xiang, ${ }^{3}$ P. Bian, ${ }^{4}$ X. Ding, ${ }^{1}$ A. Tseng,,${ }^{1}$ H. L. Hu, ${ }^{1}$ H. M. Fan, ${ }^{5}$ R. K. Zheng, ${ }^{4}$ \\ X. L. Wang, ${ }^{3}$ Z. Salman, ${ }^{2}$ T. Prokscha, ${ }^{2}$ K. Suzuki, ${ }^{6}$ R. Liu, ${ }^{7}$ S. Li, ${ }^{1}$ E. Morenzoni, ${ }^{2}$ and J. B. Yi ${ }^{1, *}$ \\ ${ }^{1}$ School of Materials Science and Engineering, UNSW, Sydney, NSW 2052, Australia \\ ${ }^{2}$ Laboratory for Muon Spin Spectroscopy, Paul Scherrer Institut, 5232 Villigen, Switzerland \\ ${ }^{3}$ Institute for Superconducting and Electronic Materials, University of Wollongong, Wollongong, New South Wales 2522, Australia \\ ${ }^{4}$ School of Physics, The University of Sydney, NSW 2006, Australia \\ ${ }^{5}$ College of Chemistry and Materials Science, Northwest University, Xi'an, Shaanxi 710069, China \\ ${ }^{6}$ Department of Materials Science and Engineering, Monash University, 3800 Victoria, Australia \\ ${ }^{7}$ SIMS Facility, Office of the Deputy-Vice Chancellor (Research and Development), Western Sydney University, \\ Locked Bag 1797, Penrith, New South Wales 2751, Australia
}

(Received 13 March 2017; published 18 September 2017)

\begin{abstract}
Co doped $\mathrm{ZnO}$ films have been deposited by a laser-molecular beam epitaxy system. X-ray diffraction and UV spectra analysis show that Co effectively substitutes the $\mathrm{Zn}$ site. Transmission electron microscopy (TEM) and secondary ion mass spectroscopy analysis indicate that there are no clusters. Co dopants are uniformly distributed in $\mathrm{ZnO}$ film. Ferromagnetic ordering is observed in all samples deposited under an oxygen partial pressure, $\mathrm{PO}_{2}=10^{-3}, 10^{-5}$, and $10^{-7}$ torr, respectively. However, the magnetization of $\mathrm{PO}_{2}=10^{-3}$ and $10^{-5}$ is very small at room temperature. At low temperature, the ferromagnetic ordering is enhanced. Muon spin relaxation $(\mu \mathrm{SR})$ measurements confirm the ferromagnetism in all samples, and the results are consistent with magnetization measurements. From $\mu$ SR and TEM analysis, the film deposited under $\mathrm{PO}_{2}=10^{-7}$ torr shows intrinsic ferromagnetism. However, the volume fraction of the ferromagnetism phase is approximately $70 \%$, suggesting that the ferromagnetism is not carrier mediated. Resistivity versus temperature measurements indicate Efros variable range hopping dominates the conductivity. From the above results, we can confirm that a bound magnetic polaron is the origin of the ferromagnetism.
\end{abstract}

DOI: 10.1103/PhysRevB.96.104423

\section{INTRODUCTION}

Spintronics is known as the technology which combines charge and spin degree of freedom of electrons. It has the advantage that devices can be operated with high speed and low power consumption [1-3]. Diluted magnetic semiconductors (DMSs) have been widely investigated in the past decades due to the promising properties that could be utilized in spintronics, which is possible to integrate the spin transport into the conventional electronic devices [1]. The ideal DMS is to dope a small amount of magnetic element into a semiconductor matrix and generate long range ferromagnetic ordering via a carrier-mediated mechanism that could make this material suitable for spintronic devices [4]. The early research on DMS showed that III-V based DMS such as Mn-doped GaAs exhibited carrier-mediated ferromagnetism [4-7]. Mean field theory based on the Zener model has been used to explain the emergence of ferromagnetism [4]. However, its low Curie temperature makes it not suitable for practical applications [4,8]. Dietl et al. predicted that DMSs based on wide band gap semiconductors (i.e., $\mathrm{ZnO}$ ) are likely to show room temperature ferromagnetism when doping by about $5 \% \mathrm{Mn}$ [4]. Wide gap semiconductors doped with transition metal elements such as, $\mathrm{Fe}, \mathrm{Cr}, \mathrm{Mn}$, and $\mathrm{Co}$ have been widely reported to exhibit ferromagnetism at room temperature thereafter [9-17]. However, the underlying coupling mechanism resulting in a ferromagnetic ground state is still under debate. In some cases, intrinsic ferromagnetism has been claimed in

*Corresponding author: jiabao.yi@unsw.edu.au oxide based DMSs [18], whereas, in some other DMSs, the ferromagnetism is not carrier-mediated and therefore cannot be explained using the Zener model based mean field theory since the samples show ferromagnetic ordering in an insulating state [19]. Therefore, the model of bound magnetic polarons has been employed for the explanation [20]. In some other cases, clusters, secondary phases, or interface structures have been claimed to be as the origin of the ferromagnetism in these oxide based DMSs [21-27]. In addition, it was also reported that transition oxide semiconductors doped with transition metals with "perfect" structures, i.e., without defects did not show ferromagnetism [28,29], indicating that defects play an important role in the formation of ferromagnetic ordering. Furthermore, ferromagnetism has been discovered in pure oxide semiconductors and nonmagnetic element doped oxide semiconductors, suggesting that defects themselves could be ferromagnetic under certain circumstances [30-39]. Co doped $\mathrm{ZnO}$, one of the typical examples for oxide based DMSs, has been studied extensively. Similar to other oxide based DMSs, ferromagnetism and paramagnetism are both reported in this system. Li et al. grew high quality $\mathrm{Co}-\mathrm{ZnO}$ thin films using molecular beam epitaxy (MBE). They found that at low doping concentration of $\mathrm{Co}$, only paramagnetism could be observed when the film was grown "perfectly," i.e., without defects [40]. However, ferromagnetic ordering can be observed when $\mathrm{Al}$ or Ga was co-doped with Co. Detailed studies indicate that the carrier concentration has been significantly increased after the Co doping, pointing to the important role of charge carriers in the formation of ferromagnetism. Hence, the ferromagnetic state may be due to the carrier mediation. However, in some reports, the formation of bound magnetic polarons were 
claimed to be attributed to the origin of the ferromagnetism in Co doped $\mathrm{ZnO}[41,42]$. In addition, as mentioned before, defects have been considered as one of the major contributions to the ferromagnetism. Transmission electron microscopy (TEM) analysis and energy dispersive $\mathrm{x}$-ray spectroscopy (EDS)/electron energy loss spectroscopy (EELs) mapping are typically used to obtain structure information and to provide evidence of intrinsic ferromagnetism, i.e., absence of dopant clusters and uniformity of dopant distribution. However, the information regarding magnetic spatial uniformity cannot be obtained using these techniques. In addition, it is still not clear whether intrinsic ferromagnetism and cluster induced ferromagnetism can co-exist in one system simultaneously. Muon spin relaxation ( $\mu \mathrm{SR})$ is a unique technique that can probe the local magnetic field in the samples, thus obtaining the information of the volume fraction of magnetic phases [43]. In addition, the tuneable energy of a low-energy muon beam (from 0.5 to $30 \mathrm{keV}$ ), allowing us to adjust the stopping depth from a few nanometer to $200 \mathrm{~nm}$, is able to identify the uniformity of magnetic profile [44]. This technique has been successfully applied to a variety of systems such as (Ga,Mn)As [45] or oxide heterostructures [46-49]. Magnetic uniformity has been reported in $\mathrm{Mn}$ doped GaAs magnetic semiconductors, confirming the intrinsic and carrier-mediated ferromagnetism [45]. However, the spatially magnetic uniformity on oxide based DMSs is rarely reported.

In this work, we perform low energy muon spin relaxation (LE- $\mu \mathrm{SR}$ ) to investigate the effects of oxygen partial pressure on the magnetic properties of $10 \%$ Co-doped $\mathrm{ZnO}$ thin films deposited by laser molecular beam epitaxy (LMBE). Hysteresis loop measurements show that pure $\mathrm{ZnO}$ thin film are close to being diamagnetic. However, ferromagnetic ordering is observed in all Co-doped $\mathrm{ZnO}$ thin films and the magnetization increases with decreasing oxygen partial pressure. TEM-EDS mapping suggests that no Co clusters exist in the Co-doped $\mathrm{ZnO}$ films, while $\mathrm{x}$-ray photoelectron spectroscopy (XPS), UV, and secondary ion mass spectroscopy (SIMS) analysis show that the Co ions are in the valance state of $2+$ and successfully substitute $\mathrm{Zn}$ ions. $\mu \mathrm{SR}$ results show that the magnetic volume fraction is lower than $70 \%$, suggesting that carrier mediation may not be as the origin of ferromagnetism. The bound magnetic polaron model can explain the observed phenomena $[41,42]$.

\section{EXPERIMENT PROCEDURE}

\section{A. Target sintering}

$\mathrm{Co}_{3} \mathrm{O}_{4}$ and $\mathrm{ZnO}$ (Sigma Aldrich, 99.99\%) powders were mixed together with an atomic ratio of $\mathrm{Co}: \mathrm{Zn}=1: 10$. The sufficiently mixed powders were pressed into pellets and sintered in a furnace at $1100{ }^{\circ} \mathrm{C}$ for ten hours. XRD analysis confirmed that the sintered target has a pure $\mathrm{ZnO}$ phase without any impurity phase. Similarly, a pure $\mathrm{ZnO}$ target was sintered using pure $\mathrm{ZnO}$ powders following the same procedure.

\section{B. Preparation of $\mathrm{ZnO}$ and Co-doped $\mathrm{ZnO}$ films}

$\mathrm{ZnO}$ thin films were fabricated by LMBE at $500{ }^{\circ} \mathrm{C}$ under different oxygen partial pressures varying from $10^{-3}, 10^{-5}$, to $10^{-7}$ torr. Both $\mathrm{ZnO}$ and $10 \%$ Co-doped $\mathrm{ZnO}$ thin films were grown on (0001) quartz substrates. A pulsed KrF excimer laser, operated with the energy and frequency of $200 \mathrm{~mJ}$ and $1 \mathrm{~Hz}$, respectively, was used for the film deposition. Each deposition was kept at 2000 pulses. The deposited films are approximately $50 \mathrm{~nm}$ in thickness.

\section{Materials characterizations}

The crystal structures of the thin films were analyzed by $\mathrm{X}$-ray diffractometery (XRD, PANalytical Empyrean thin-film $\mathrm{X}$-ray diffraction system, $\mathrm{Cu} \mathrm{K} \alpha$ radiation). The morphologies and structures of the samples were examined by TEM (Phillips CM200). Raman spectroscopy (Renishaw inVia Raman microscope equipped with a $325 \mathrm{~nm} \mathrm{He}-\mathrm{Cd}$ ion laser and fitted with a diffraction grating of 1800 lines $/ \mathrm{mm}$ ) was used for determining the crystalline phase and point defects. UV spectroscopy analysis (PerkinElmer UV-Visible Spectrometer) was carried out to study the electronic transition in Co-doped $\mathrm{ZnO}$. The electronic structure was characterized by $\mathrm{x}$-ray photoelectron spectroscopy (XPS, ThermoScientific ESCLAB 250i). The depth profiles of elemental distribution in the thin film samples were obtained by a time of flight secondary ion mass spectrometer (TOFSIMS, ION-TOF TOF.SIMS). A magnetic property measurement system and physical property measurement system (PPMS) (Quantum Design, USA) were used for measuring M-H loops and transport properties.

\section{Muon spin relaxation measurement}

The $\mu \mathrm{SR}$ measurements were performed at the $\mu \mathrm{E} 4$ beam line [50] of the Swiss Muon Source, Paul Scherrer Institute, Switzerland. The measurements are performed using the lowenergy $\mu \mathrm{SR}$ technique, where an intense high-energy beam of muons is moderated in a solid Ar film. A small fraction of the incident muons emerge from the argon surface at low energy $(10 \mathrm{eV})$. These are subsequently accelerated to $15 \mathrm{keV}$ and transported to the sample chamber and $\mu$ SR spectrometer. The square samples $(20 \mathrm{~mm} \times 20 \mathrm{~mm})$ are mounted onto a $\mathrm{Ni}$-coated Al plate which is electrically isolated from the cold finger of the cryostat and biased to a high voltage ranging from -12.5 to $12.5 \mathrm{kV}$. This allows the implantation energy of the muons to be adjusted between about 1.5 and $26.5 \mathrm{keV}$. We performed the measurements with and without magnetic field. A transverse field (TF) of 30 Oe is applied perpendicular to the initial muon spin polarization and the substrate surfaces, which are used to determine the magnetic volume fraction as a function of temperature. For the zero magnetic field (ZF) measurements, the stray magnetic field at the sample is less than 0.1 Oe.

\section{RESULTS AND DISCUSSION:}

XRD measurements were carried out to examine the crystallinity and to identify the different crystalline phases in the films (Fig. 1). The results show the wurtzite phase of $\mathrm{ZnO}$ with its $c$-axis grown normal to the substrate surface. Only the (002) and (004) peaks of $\mathrm{ZnO}$ are present which indicates the high quality and epitaxial growth. No secondary phases are detected. In addition, it can be seen that with the decrease in the oxygen partial pressure, the peak (002) has a slight shift to the lower angle. It may be due to the stress induced by film growth 


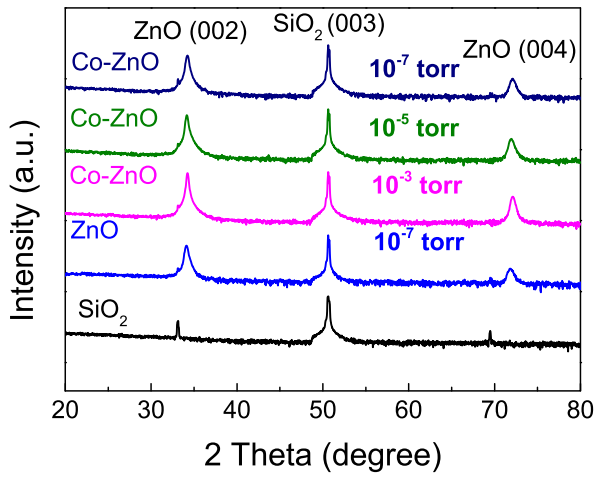

FIG. 1. XRD spectra of pure $\mathrm{ZnO}$ and $\mathrm{Co}$ doped $\mathrm{ZnO}$ films deposited under different oxygen partial pressures.

or some Co ions enter the interstitial site. High resolution TEM (HRTEM) images confirm the $c$-axis growth as shown in Figs. 2(a)-2(c). However, two orientations with a common $c$ axis are observed in the images with two sets of lattice fringes. The corresponding selected area electron diffraction (SAED) patterns are presented in the insets of Figs. 2(a)-2(c). The spots labeled in blue and yellow belong to the $\mathrm{ZnO}[11 \overline{2} 0]$ and $\mathrm{ZnO}$ [1010] zone axes, respectively, which suggest that the two orientation domains are twisted by $30^{\circ}$. The formation of twisted domains is caused by the competition between the local interface energy and epitaxial strain energy [51-53]. This may attribute to the relatively low growth temperature. Hence, there is insufficient energy to overcome the energy barrier. Similar phenomena were reported in $\mathrm{ZnO}$ thin films grown by pulsed laser deposition [52,54]. The lattice in [0002] zone axis increases with the decreasing in oxygen partial pressure, supporting that Co ions may enter interstitial sites due to relatively high doping concentration, in consistence with XRD results. The images obtained by EDS mapping attached in the TEM system, as shown in Figs. 2(d)-2(f), reveal the uniform distribution of $\mathrm{Co}$ atoms in $\mathrm{ZnO}$ films. No evidence of $\mathrm{Co}$ clusters is observed in all samples. From the EDS mapping, it can be seen that the thickness of the films for $\mathrm{PO}_{2}=10^{-3}$, $10^{-5}, 10^{-7}$ torr is 65,60 , and $45 \mathrm{~nm}$, respectively, indicating that oxygen partial pressure can affect the film deposition rate.

Multiphonon LO modes are observed in Raman spectra with a $325 \mathrm{~nm} \mathrm{He}-\mathrm{Cd}$ laser as the excitation source [Fig. 3(a)], which are detected at $578 \mathrm{~cm}^{-1}$ (LO), $1163 \mathrm{~cm}^{-1}$ (2LO), and $1742 \mathrm{~cm}^{-1}$ (3LO). It is noted that the peak intensity is significantly increased with the addition of Co dopants. The enhancement of the multiphonon scattering may attribute to the nature of $\mathrm{Co}$ atoms incorporated in the $\mathrm{ZnO}$ thin films and the intrinsic point defect scattering, such as oxygen and zinc vacancies, generated by the Co doping [55].

The optical absorption spectra were measured to study the electronic transition. Three absorbance peaks at 568, 612, and $661 \mathrm{~nm}$ are observed in the absorbance spectra of Co-doped $\mathrm{ZnO}$ thin films [Fig. 3(b)], which are attributed to the d-d electronic transitions of tetrahedrally coordinated $\mathrm{Co}^{2+}$ due to the ${ }^{4} \mathrm{~A} 2(\mathrm{~F}) \rightarrow{ }^{2} \mathrm{~A} 1(\mathrm{G}),{ }^{4} \mathrm{~A} 2(\mathrm{~F}) \rightarrow{ }^{4} \mathrm{~T} 1(\mathrm{P})$, and ${ }^{4} \mathrm{~A} 2(\mathrm{~F}) \rightarrow$ ${ }^{2} \mathrm{E}(\mathrm{G})$ transitions, respectively, involving crystal-field-split $3 \mathrm{~d}$ levels of $\mathrm{Co}^{2+}$ ions substitution on $\mathrm{Zn}$ sites [56-59]. This observation gives clear evidence that Co ions have entered the $\mathrm{ZnO}$ lattice and suggests that there is no formation of $\mathrm{Co}$ clusters. These results are consistent with the EDS mapping. In addition, SIMS was performed to study Co ions distribution. All the samples show uniform distribution of $\mathrm{Co}^{2+}$ ions. A typical spectrum of a $\mathrm{PO}_{2}=10^{-7}$ torr sample is shown in Fig. 3(c). The increase in $\mathrm{Zn}^{2+}$ and $\mathrm{Co}^{2+}$ signal at the interface is caused by the changes in the ion yields at the interface, which is often observed in SIMS measurements [60]. There is a slight difference of thickness between EDS and SIMS measurement. It is due to the measurement error of the depth of the etched hole produced by SIMS measurement. XPS analysis was performed to further confirm the valence states and the oxygen vacancy states of the samples. The separation of Co $2 \mathrm{p}^{3 / 2}$ and Co $2 \mathrm{p}^{1 / 2}$ shown in Fig. 4(d) is approximately $16 \mathrm{eV}$, indicating the valance state of Co ion is $2+$. Figures 4(a)-4(c) show the spectra of $\mathrm{O} 1 \mathrm{~s}$ edge for the $\mathrm{PO}_{2}=10^{-3}, 10^{-5}$, and (a)

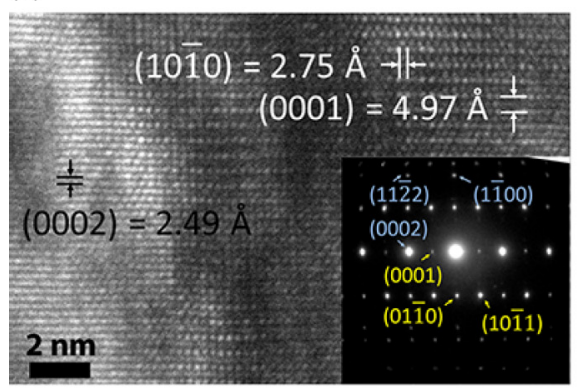

(d)

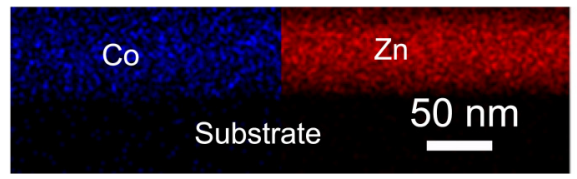

(b)

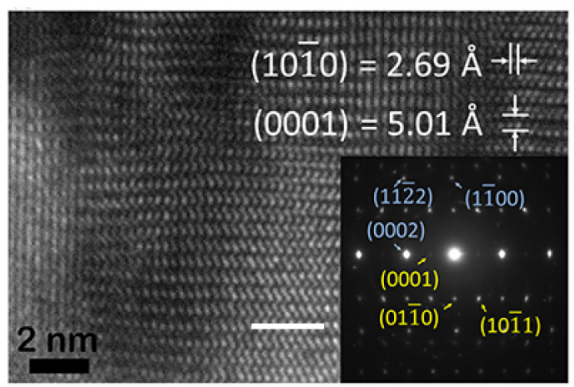

(e)

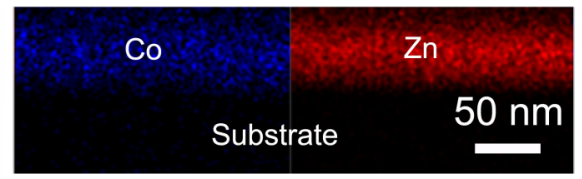

(c)

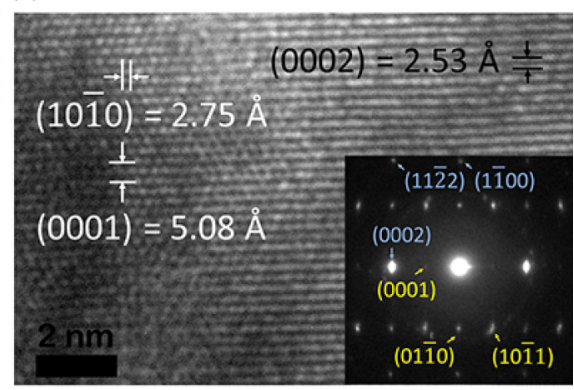

(f)

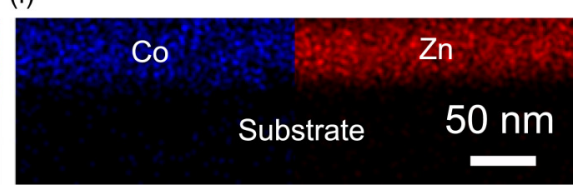

FIG. 2. High resolution TEM micrographs of $10 \%$ Co-doped $\mathrm{ZnO}$ thin films prepared at different oxygen partial pressures (a) $10^{-3}$ torr, (b) $10^{-5}$ torr, (c) $10^{-7}$ torr; insets show the SAED patterns where the spots labeled in yellow and blue belong to the $\mathrm{ZnO}[1 \overline{1} 0]$ and $\mathrm{ZnO}[11 \overline{2} 0$ ] zone axes in a relative large area. EDS mapping images of Co and $\mathrm{Zn}$ elements in the samples deposited at (d) $10^{-3}$ torr, (e) $10^{-5}$ torr, (f) $10^{-7}$ torr. 

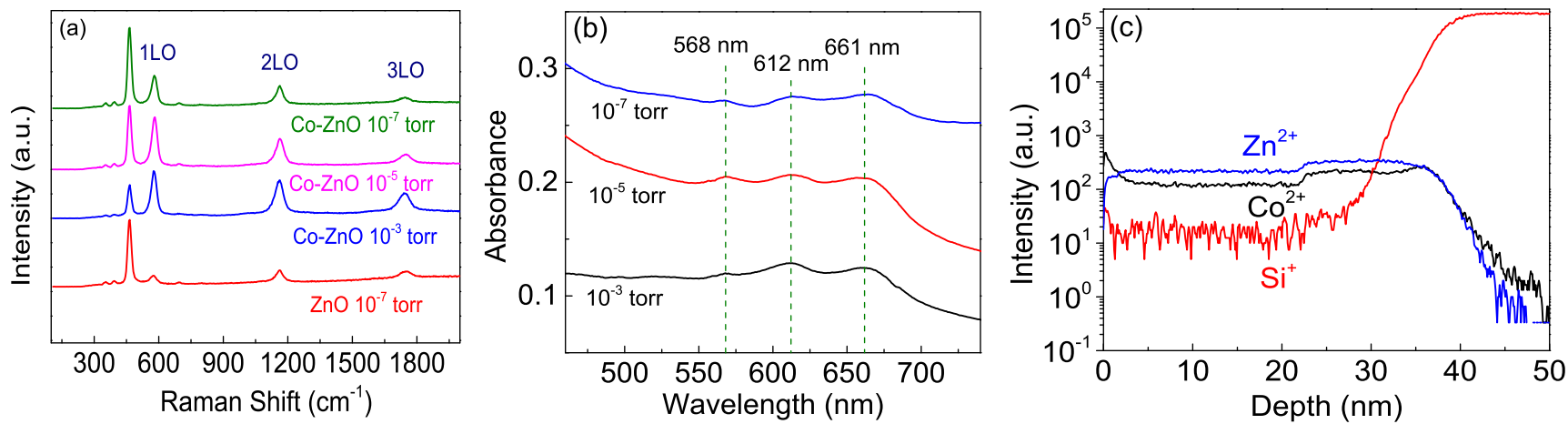

FIG. 3. (a) Raman spectra of $\mathrm{ZnO}$ and Co-doped $\mathrm{ZnO}$ thin films excited by $325 \mathrm{~nm}$ He-Cd laser. (b) UV absorbance spectra of Co doped $\mathrm{ZnO}$ films. (c) SIMS spectra of Co doped $\mathrm{ZnO}$ film deposited under $\mathrm{PO}_{2}=10^{-7}$ torr.

$10^{-7}$ torr samples, respectively. With careful fitting, it can be seen that the oxygen contribution to $\mathrm{Zn}-\mathrm{O}$ bond is suppressed with decreasing the oxygen partial pressure, suggesting the increase in vacancy concentration of oxygen.

The $\mathrm{M}-\mathrm{H}$ loops of Co-doped $\mathrm{ZnO}$ films are shown in Fig. 5. Pure $\mathrm{ZnO}$ film deposited at $\mathrm{PO}_{2}=10^{-7}$ torr shows a very small magnetization at both 5 ( $\mathrm{K}$ and room temperature $\left(2 \mathrm{emu} / \mathrm{cm}^{3}\right)$. The room temperature hysteresis loops are observed in all Co-doped $\mathrm{ZnO}$ films. The saturation magnetization at room temperature of Co-doped $\mathrm{ZnO}$ films deposited at $\mathrm{PO}_{2}=10^{-3}, 10^{-5}$, and $10^{-7}$ torr is $2.5,10$, and $35 \mathrm{emu} / \mathrm{cm}^{3}$, respectively, which increases with decreasing oxygen partial pressure. It should be noted that the resolution of superconducting quantum interference devices (SQUID) for this kind of film measurement is around $1 \mathrm{emu} / \mathrm{cm}^{3}$. Hence, the measured magnetization of the pure $\mathrm{ZnO}$ film and the $\mathrm{Co}$ doped $\mathrm{ZnO}$ film deposited under $\mathrm{PO}_{2}=10^{-3}$ torr is not very accurate. At $5 \mathrm{~K}$, the saturation magnetization of the samples deposited at $\mathrm{PO}_{2}=10^{-3}, 10^{-5}$, and $10^{-7}$ torr increases to 6.5 , 16 , and $60 \mathrm{emu} / \mathrm{cm}^{3}$, respectively. A significant increase in the coercivity of Co-doped $\mathrm{ZnO}$ film deposited at $\mathrm{PO}_{2}=10^{-7}$ torr is also observed.

The high magnetization of the $\mathrm{PO}_{2}=10^{-7}$ torr sample may be due to the high concentration of carriers which would imply
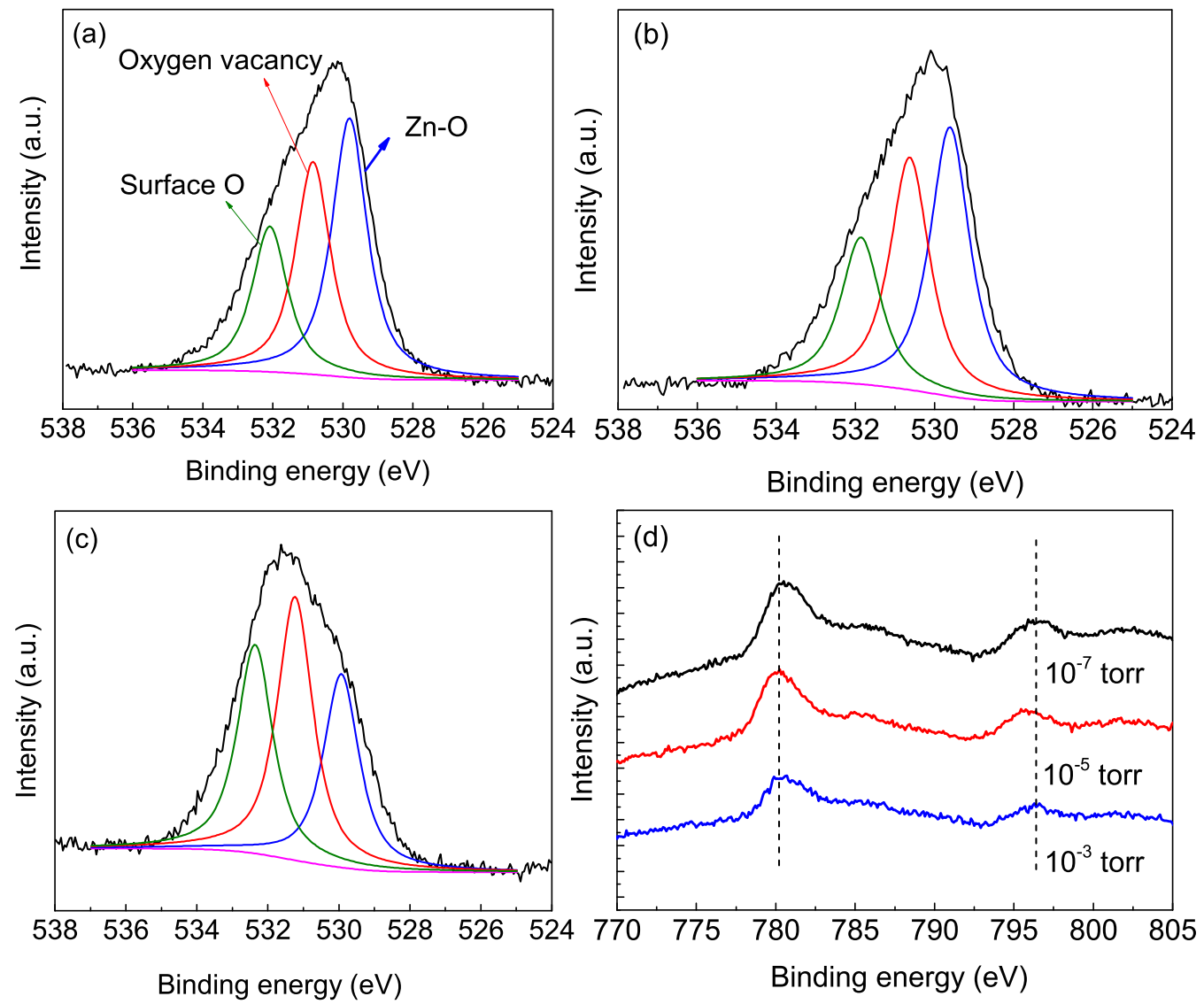

FIG. 4. XPS spectra of the $\mathrm{O} 1 \mathrm{~s}$ edge of (a) $\mathrm{PO}_{2}=10^{-3}$, (b) $\mathrm{PO}_{2}=10^{-5}$, and (c) $\mathrm{PO}_{2}=10^{-7}$ torr. (d) XPS spectra of the Co $2 \mathrm{p}$ core level in Co-doped $\mathrm{ZnO}$ thin films. 

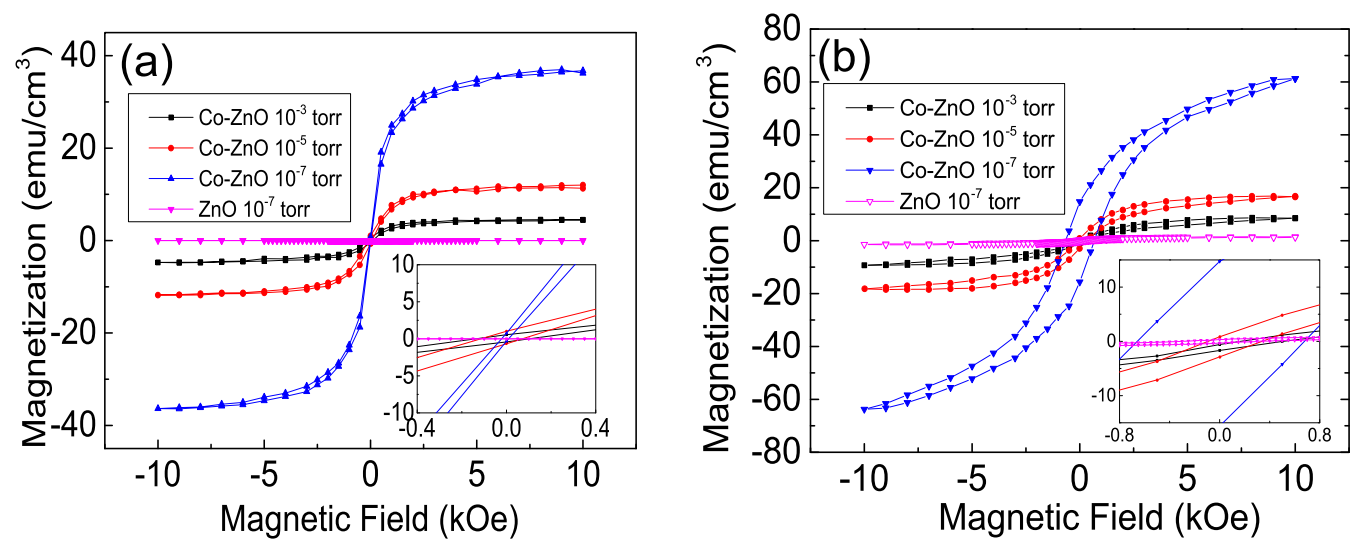

FIG. 5. M-H loops of $10 \%$ Co-doped $\mathrm{ZnO}$ thin films with different oxygen partial pressures measured at (a) room temperature and (b) $5 \mathrm{~K}$.

that carrier mediation may be the origin of the enhancement of the ferromagnetism. To support whether the ferromagnetism is carrier mediated or not, we performed annealing of the $\mathrm{PO}_{2}=$ $10^{-3}$ torr sample under an oxygen partial pressure of $10^{-7}$ torr at $500{ }^{\circ} \mathrm{C}$ for $1 \mathrm{~h}$. The saturation magnetization of the sample is significantly enhanced, reaching $10.65 \mathrm{emu} / \mathrm{cm}^{3}$ at $300 \mathrm{~K}$ and $22 \mathrm{emu} / \mathrm{cm}^{3}$ at $5 \mathrm{~K}$, supporting that the ferromagnetism is carrier mediated since oxygen vacancies induced by annealing lead to carriers to mediate the ferromagnetism. In order to study the origin of ferromagnetism, resistivity versus temperature curves were taken using PPMS, as shown in Fig. 6. The resistivity is $0.18 \Omega \mathrm{cm}$ and $0.026 \Omega \mathrm{cm}$ at room temperature for $\mathrm{PO}_{2}=10^{-5}$ and $10^{-7}$ torr samples, respectively. It should be noted that the resistivity of $\mathrm{PO}_{2}=10^{-3}$ torr samples at low temperature is beyond our system limitation and cannot be measured. From Hall measurement, the corresponding carrier concentrations for the $\mathrm{PO}_{2}=10^{-5}$ and $10^{-7}$ torr sample at $300 \mathrm{~K}$ are $3.5 \times 10^{18}$ and $7.6 \times 10^{20} \mathrm{~cm}^{-3}$, respectively. The significantly increased resistivity of both samples at low temperature indicates the semiconducting nature [Fig. 6(a)]. In order to understand the conduction mechanism, we fitted the curves with $\ln \rho$ versus $\mathrm{T}^{-1 / 2}$ and $\mathrm{T}^{-1 / 4}$, as shown in Fig. 6. $\ln \rho$ depends linearly on $\mathrm{T}^{-1 / 2}$, suggesting that the Efros variable range hopping (VRH) may be the dominant conduction mechanism, meaning that Coulomb interactions between carriers dominate the electrical transport [61]. The hopping resistivity can be described as $\ln \rho / \rho_{0}=\left(\mathrm{T} / \mathrm{T}_{\text {Efros }}\right)^{-1 / 2}[19,61,62]$, where $\mathrm{T}_{\text {Efros }}=8 \mathrm{e}^{2} / \varepsilon_{r} \mathrm{k}_{B} \xi, \rho_{0}$ is the resistivity coefficient, e is the electron charge, $\varepsilon_{r}$ is the dielectric constant [20], $k_{B}$ is the Boltzmann constant, and $\xi$ is the localization length of carriers near the Fermi level. The VRH conduction mechanism and the small change of saturation magnetization at room and low temperatures suggest that bound magnetic polarons may be the origin of the ferromagnetism.

Intrinsic ferromagnetism is essential for the applications in spintronics devices. It is known that magnetic clusters are likely to be formed in thin films prepared at low oxygen partial pressure, which were claimed to be the origin of ferromagnetism [27]. XRD and TEM-EDS mapping indicate that there is no Co clusters in the samples. In addition, UV and XPS analysis have shown that the Co ions have entered the $\mathrm{Zn}$ lattice sites with +2 valence state. This indicates the structural uniformity of our samples. To elucidate the nature of ferromagnetism in our samples, we performed low energy $\mu \mathrm{SR}$ measurements. These measurement allow us to investigate the magnetic uniformity and to identify the volume fraction of the ferromagnetic phase $[45,65]$. In this work, we performed a depth profile with a range of tuneable muon beam energies from 1 to $20 \mathrm{keV}$ for comparing the thin film and substrate $\mu \mathrm{SR}$ signals, respectively. The calculated muon stopping profiles for various implantation energies are shown in Fig. 7.

The asymmetry spectrum corresponds to the time evolution of the muon spin polarization. Figure 8(a) shows the asymmetry spectra measured in a transverse field (TF) of 30 Oe applied perpendicular to the sample surface at $5 \mathrm{~K}$. All the $\mu \mathrm{SR}$ data were analyzed using the MUSRFIT data analysis framework for
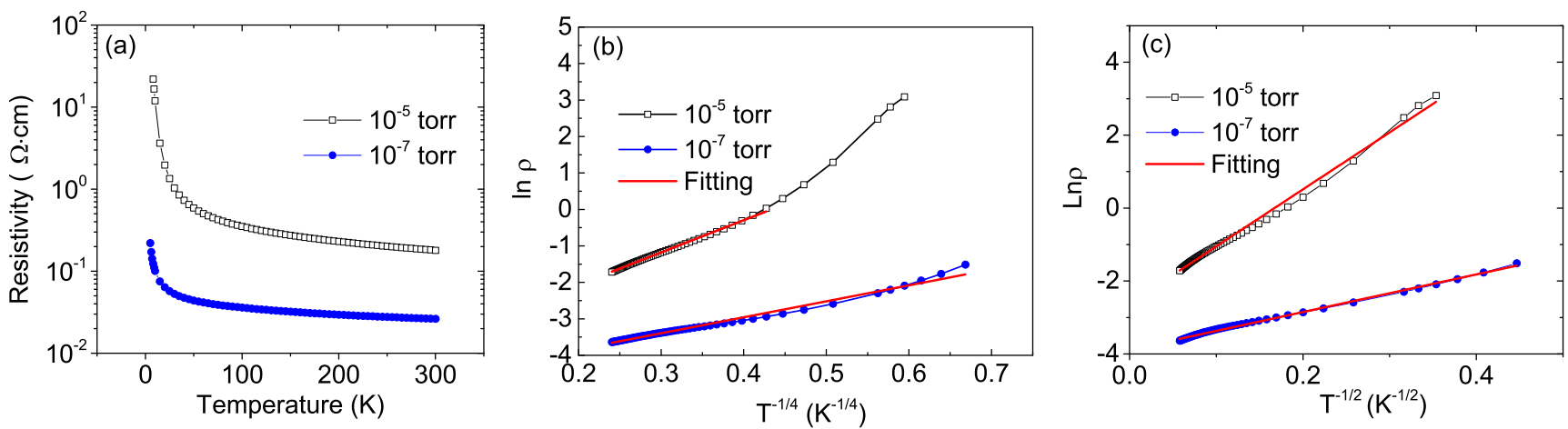

FIG. 6. Resitivity-versus-temperature curves of Co doped $\mathrm{ZnO}$ films deposited under $\mathrm{PO}_{2}=10^{-5}$ and $10^{-7}$ torr. (a) Resistivity, $\rho$ versus T. (b) $\ln \rho$ versus $\mathrm{T}^{-1 / 4}$. (c) $\ln \rho$ versus $\mathrm{T}^{-1 / 2}$. 


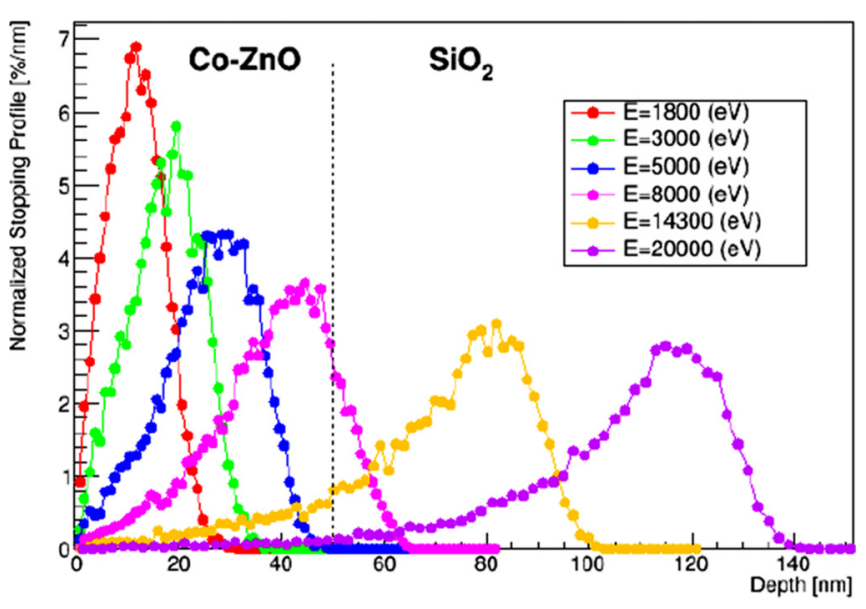

FIG. 7. Muon stopping profiles for various implantation energies E calculated with the Monte-Carlo program TRIMSP [63,64].

$\mu \mathrm{SR}$ [66]. The data was fitted with a cosine function multiplied by a exponential with a damping rate $\lambda_{s}$ taking into account the field inhomogeneities,

$$
P(t)=A \cos \left(\gamma_{\mu} B t+\phi\right) \mathrm{e}^{-\lambda_{s} t} .
$$

The amplitude of the asymmetry, A, is a measure of the nonmagnetic volume fraction, while $\lambda_{s}$ is the damping due to magnetic field inhomogeneities produced by the ferromagnetic regions in the sample. By comparing $\mathrm{A}$ to the the reference asymmetry in a nonmagnetic sample of similar geometry, we can deduce the nonmagnetic volume fraction of the magnetic films.

The asymmetry spectrum of the undoped $\mathrm{ZnO}$ reference sample shows a weak depolarization, consistent with the presence of weak nuclear dipolar fields from ${ }^{67} \mathrm{Zn}$ nuclei with low abundance. This indicates that there is no ferromagnetic phase in the sample. In contrast, the Co-doped $\mathrm{ZnO}$ samples deposited at oxygen partial pressures varied from $10^{-3}$ to $10^{-7}$ torr show a reduced initial polarization, because muons stopping in magnetic regions experience large internal magnetic fields, causing a very quick loss of polarization ("missing fraction"). The remaining precession signal still shows a relatively weak depolarization rate, indicating that this fraction originates from muons stopping in regions without magnetic order. Typical asymmetry spectra measured in zero field $(\mathrm{ZF})$ are presented in Fig. 8(b). The ZF spectra in strongly magnetic samples $\left(\mathrm{PO}_{2}=10^{-7}\right.$ torr $)$ is fitted by a static Lorentzian Kubo-Toyabe (KT) function multiplied by an exponential

$$
P(t)=A\left[\frac{1}{3}+\frac{2}{3}(1-\Delta t) e^{-\Delta t}\right] e^{-\lambda_{e} t} .
$$

Here $\Delta$ is related to the width of the local field distribution, $\Delta B$, by $\Delta=\gamma_{\mu} \Delta B$, with $\gamma_{\mu}$ the muon gyromagnetic ratio, and $\lambda_{e}$ is the damping rate due to some slow electronic dynamics. The KT function is appropriate for a $\mu$ SR signal of randomly distributed diluted static magnetic moments. The signal in weakly magnetic samples grown at $\mathrm{PO}_{2}=10^{-3}$ and $10^{-5}$ torr is better accounted for using a simple fast-relaxing exponential plus a slowly-relaxing exponential function $\lambda_{s} \approx 0.2(1) \mu \mathrm{s}^{-1}$.

$$
P(t)=A_{s} e^{-\lambda_{s} t}+A_{f} e^{-\lambda_{f} t}
$$

The slow component represents a combination of the paramagnetic and background signal, and the nonrelaxing component of the small magnetic signal corresponding to the first term of Eq. (2). The depolarization rate of the fast component $\lambda_{f}$ is related to $\Delta$ in Eq. (2) by $\lambda_{f} \approx \frac{4}{3} \Delta$ [45].

From the fitting, the depolarization rate is very low in the reference sample of undoped $\mathrm{ZnO}$. In the Co-doped samples the depolarization rate increases with decreasing oxygen partial pressure, indicating that the ferromagnetic strength becomes stronger with decreasing oxygen partial pressure.

Figure 9(a) shows the asymmetry of the precessing signal in a transverse field of $30 \mathrm{Oe}$ as a function of temperature. The asymmetry of undoped $\mathrm{ZnO}$ sample is approximately 0.14 from $250 \mathrm{~K}$ to $5 \mathrm{~K}$. For Co-doped $\mathrm{ZnO}$ films prepared at $10^{-3}$ and $10^{-5}$ torr, the asymmetry is similar to that of the undoped reference sample at relatively high temperature. However, it decreases significantly at temperatures lower than $50 \mathrm{~K}$, which can be attributed to the appearance of regions with ferromagnetic order with large internal magnetic fields, causing a significant reduction of the 30 Oe precession

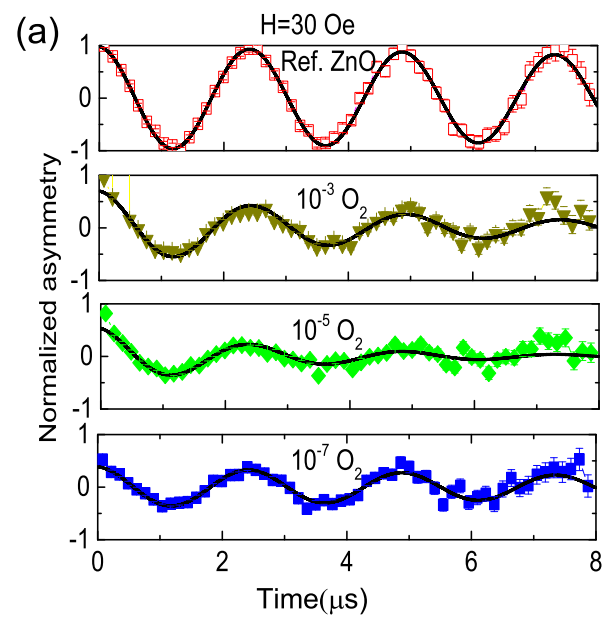

\section{(b)}

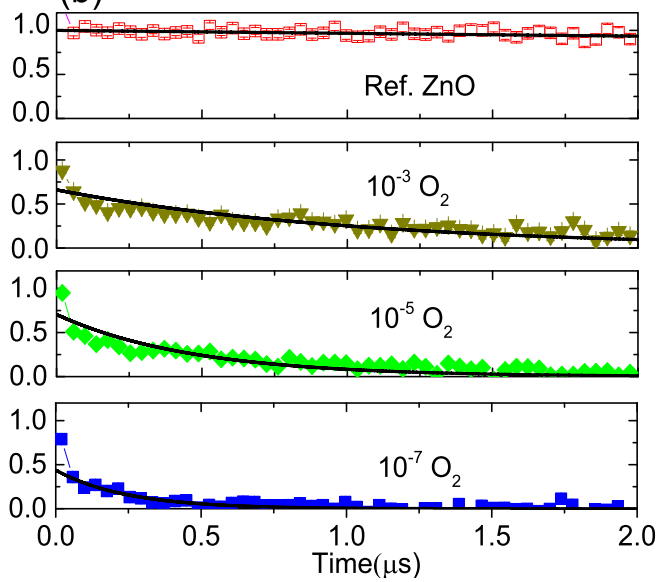

FIG. 8. Normalized low energy ( $5 \mathrm{keV}) \mu \mathrm{SR}$ asymmetry spectra of Co-doped $\mathrm{ZnO}$ films measured in (a) a transverse field of 30 Oe perpendicular to the sample surface and (b) at zero field. 

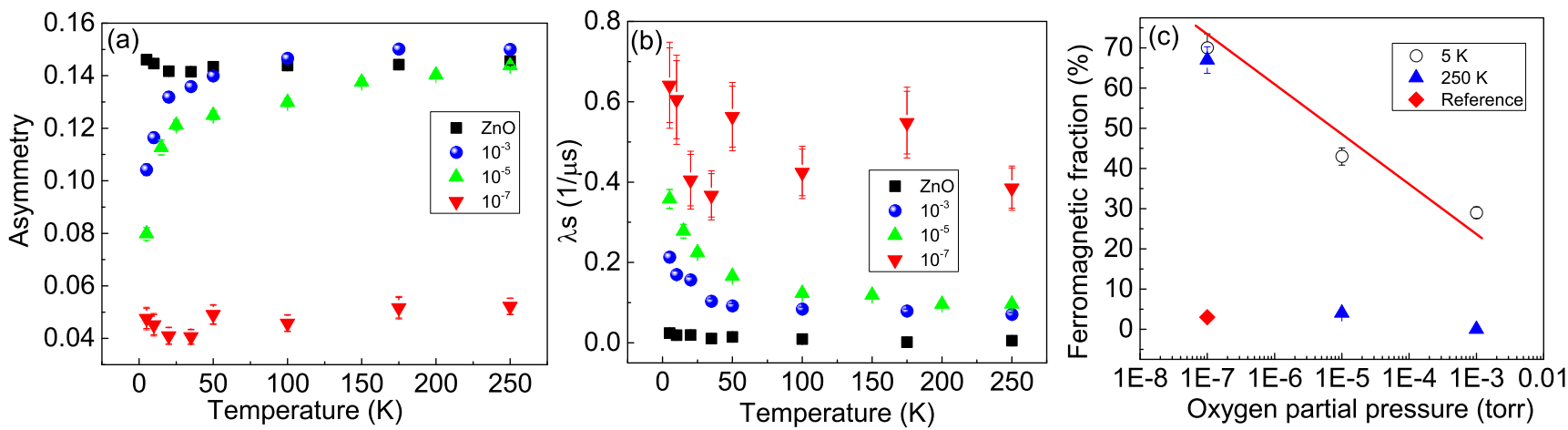

FIG. 9. Temperature dependence of (a) the asymmetry, (b) the relaxation rate $\lambda_{s}$ in pure and Co-doped $\mathrm{ZnO}$ films. (c) Volume fraction of the ferromagnetic phase.

amplitude. It clearly shows that a large fraction of paramagnetic phase at room temperature transforms to ferromagnetic phase at low temperature. For the Co-doped $\mathrm{ZnO}$ sample deposited at $10^{-7}$ torr, the asymmetry is approximately 0.045 at all temperatures, which is significantly lower than in the other samples, indicating the presence of ferromagnetic ordering at least up to $250 \mathrm{~K}$. The transverse field depolarization rates $\lambda_{s}$ at different temperatures are shown in Fig. 9(b). For a pure $\mathrm{ZnO}$ sample, the damping rate is close to zero at all temperatures, confirming the nonmagnetic nature of the sample. For Co-doped $\mathrm{ZnO}$ samples, $\lambda_{s}$ increases with decreasing oxygen partial pressure: This increase can attribute to the development of stray fields from the ferromagnetic region in the paramagnetic regions.

Though there is variation of the damping rate in the $\mathrm{PO}_{2}=$ $10^{-7}$ torr sample, the variation as a function of temperature is relatively small. The samples prepared at $10^{-3}$ and $10^{-5}$ torr show a similar trend of $\lambda_{s}$ down to $50 \mathrm{~K}$. The increase of the damping rate at lower temperatures suggests the development of ferromagnetic order at this temperature, consistent with the trend of the asymmetry, shown in Fig. 8. As discussed previously, intrinsic ferromagnetism is of importance for the application of spintronics devices, which requires that the ferromagnetism is originated from dopant doping, not from defects and the ferromagnetism is mediated by carries. Therefore, the entire film is ferromagnetic due to carrier mediation. From the TF asymmetries, the volume fraction of the ferromagnetic phase can be calculated [45], as shown in Fig. 9(c). Pure $\mathrm{ZnO}$ has a very small fraction of ferromagnetic phase less than $3 \%$ at both 5 and $250 \mathrm{~K}$, which may be due to the defect induced ferromagnetism. The volume fraction of the ferromagnetic phase for the Co doped $\mathrm{ZnO}$ samples deposited under $\mathrm{PO}_{2}=10^{-7}, 10^{-5}, 10^{-3}$ torr is $70 \%, 43 \%$, $25 \%$, respectively, at $5 \mathrm{~K}$. At $250 \mathrm{~K}$, the fraction becomes $67 \%, 3 \%$, and $0 \%$, respectively, indicating that there is intrinsic ferromagnetism up to $250 \mathrm{~K}$ in the $\mathrm{PO}_{2}=10^{-7}$ torr sample, because at this partial oxygen pressure the magnetic volume fraction does not change within experimental errors. In addition, no clusters were observed from TEM analysis. For the $\mathrm{PO}_{2}=10^{-5}$ and $10^{-3}$ samples, there is almost no ferromagnetic order present at room temperature.

In order to investigate whether the samples have relatively uniform magnetization from surface to interface, we collected the spectra of $\mathrm{TF}-\mu \mathrm{SR}$ with different implantation energies, from which we can determine the ferromagnetic profile from the top surface to the substrate, as shown in Fig. 10. From the stopping profile calculations an implantation energy of $8 \mathrm{keV}$ corresponds to a mean implantation depth of about $45 \mathrm{~nm}$. At energies $\gtrsim 8 \mathrm{keV}$ muons start to penetrate into the $\mathrm{SiO}_{2}$ substrate where the positively charged muon predominantly captures an electron to form the neutral, hydrogenlike atom muonium. The precession frequency of a $\mu^{+}$in the neutral muonium state is typically at least two orders of magnitude larger than the $\mu^{+}$Larmor frequency, causing the observed
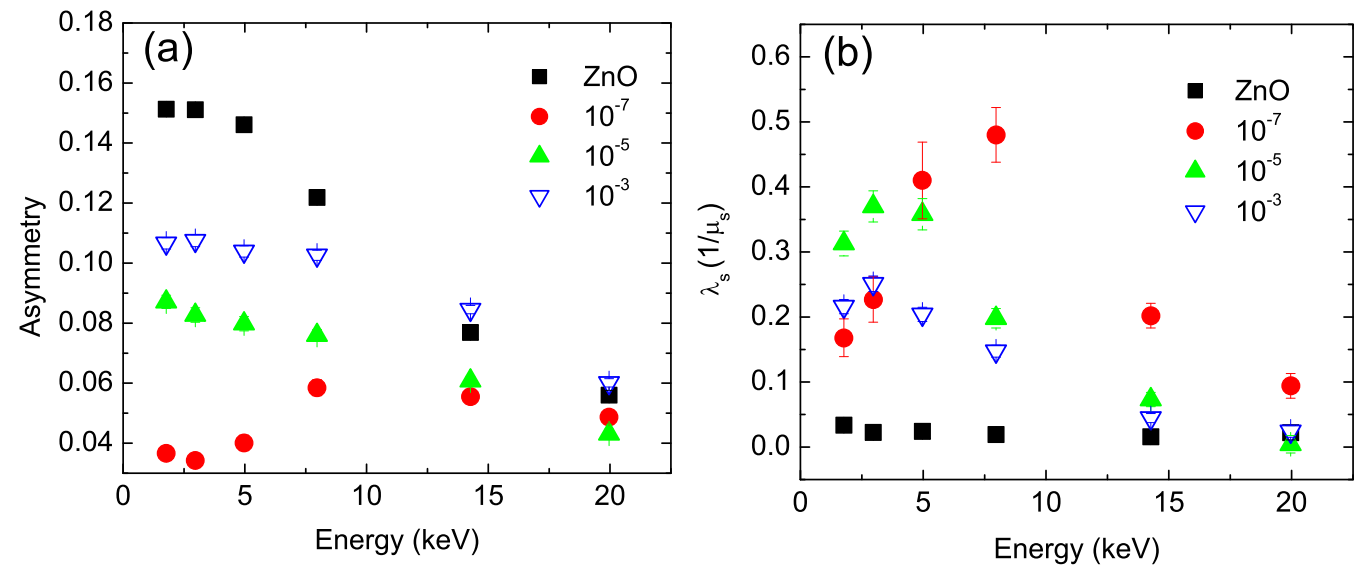

FIG. 10. Muon implantation energy dependence of (a) the asymmetry and (b) of the relaxation rate $\lambda_{s}$ in pure and Co-doped $\mathrm{ZnO}$ films. 

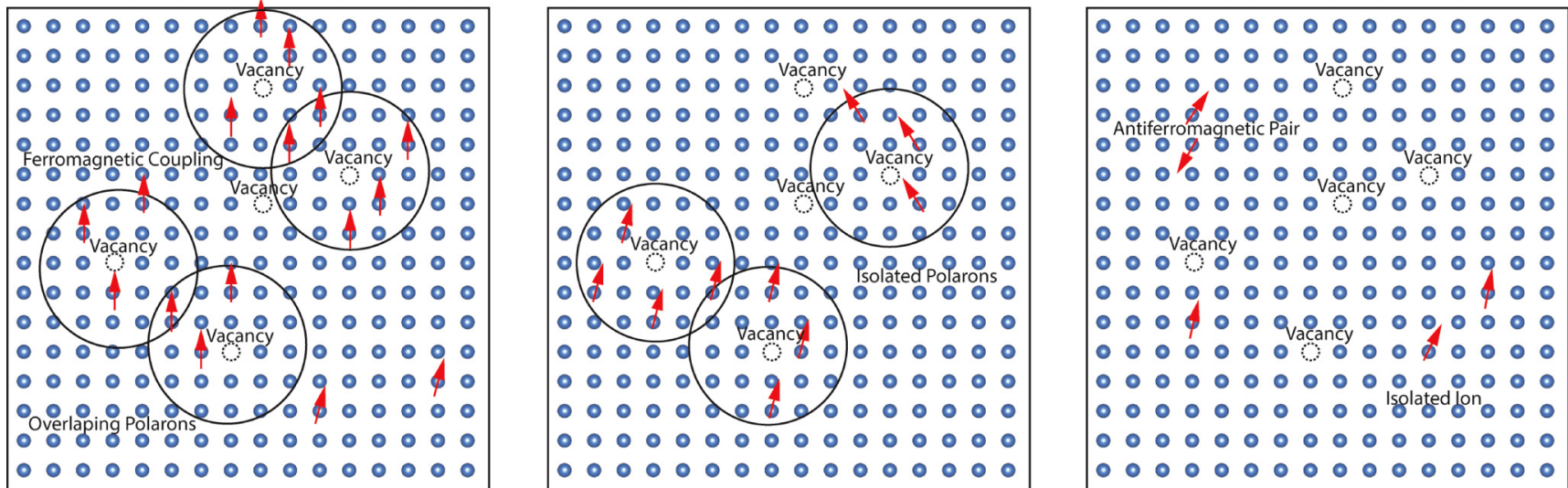

FIG. 11. A description of BMP formation and their interaction with defects (oxygen vacancies). (a) Overlap of polarons via the defect to form ferromagnetic ordering. Most part of the film is ferromagnetic. (b) A small fraction of polarons overlap via the defect interaction, leading to a small fraction of ferromagnetic phase. (c) Magnetic polarons exist in the paramagnetic phase without interaction with defects.

reduction of the asymmetry of the muon originating from Larmor precession. The fact that the asymmetry weakly depends on implantation energy for energies $\lesssim 5 \mathrm{keV}$, i.e., for muons stopping in the $\mathrm{ZnO}$ film, indicates relatively uniform ferromagnetism from surface to interface. From Fig. 10(b), it can be seen that the relaxation rate for reference sample is very low at all implantation energies. The low relaxation rate at the surface of the $\mathrm{PO}_{2}=10^{-7}$ sample suggests a more inhomogeneous magnetic state as a function of depth in this Co-ZnO film.

The XRD and TEM analysis results indicate a uniform distribution of $\mathrm{Co}$ atoms in the $\mathrm{ZnO}$ lattice without the formation of any clusters or secondary phases. In addition, XPS results show that $\mathrm{Co}$ enters the $\mathrm{ZnO}$ lattice at the $\mathrm{Zn}$ site, suggesting that the magnetic state should be uniform as well. In contrast to these findings, the $\mu \mathrm{SR}$ measurements show that the magnetic volume fractions of all samples is $\lesssim 70 \%$, demonstrating the presence of nonmagnetic regions in the films. This indicates that carrier mediation is not the origin of ferromagnetic order, though the carrier concentration from $10^{-3}$ to $10^{-7}$ increases significantly. Since the other techniques reveal a homogenous distribution of dopants and charge carriers, one would expect a $100 \%$ magnetic volume fraction in this case. The R-T curve fitting suggests that the ferromagnetism can be explained by the bound magnetic polaron theory [19], which may explain the findings of the other experimental techniques in a consistent way.

Therefore, the ferromagnetism can only be explained by bound magnetic polarons (BMP) theory, consistent with R-T curve fitting [19]. The doping of $\mathrm{Co}$ into $\mathrm{ZnO}$ forms magnetic polarons. From BMP theory [20], oxygen vacancies play important roles in the formation of ferromagnetic ordering. Oxygen vacancies can form $\mathrm{F}$ centers with trapped electrons. The orbitals of the $\mathrm{F}$ centers can interact with the d orbitals of Co dopants to form magnetic polarons. The increased concentration of magnetic polarons induces the overlapping of magnetic polarons, leading to ferromagnetic coupling. On the other hand, if there are less dopants, the magnetic polarons cannot be formed since the F center cannot be coupled with $\mathrm{d}$ orbitals. Similarly, if there is not enough oxygen vacancies, the ferromagnetic ordering cannot form either. Hence, the oxygen vacancies are very important for the formation of $\mathrm{BMP}$ in oxide based diluted magnetic semiconductors. In $\mathrm{PO}_{2}=10^{-3}$ and $10^{-5}$ samples, the oxygen vacancy concentration is not high enough. The isolated dopant or magnetic polarons cannot form ferromagnetic ordering. Therefore, there is very low volume fraction of ferromagnetic phase even at $5 \mathrm{~K}$ in the two samples. The required concentration of dopants or oxygen vacancies indicates that there is a percolation effect for BMPs [20]. For the $\mathrm{PO}_{2}=10^{-7}$ sample, the interaction of oxygen vacancies $F$ centers coupled with magnetic dopants, and the overlapping of magnetic polarons form strong ferromagnetic ordering $[19,67]$. However, the ferromagnetic phase cannot expand to the entire film (70\%). This can occur if some of the magnetic polarons do not overlap, forming some nonferromagnetic gaps between the polarons, as shown in Fig. 11(a). These nonmagnetic areas may be due to the nonuniform distribution of defects, i.e., oxygen vacancies. Therefore, in some areas, the effective coupling between vacancies and polarons cannot be formed. For the $\mathrm{PO}_{2}=10^{-5}$ and $10^{-3}$ torr samples, the concentration of defects, i.e., oxygen vacancies, is not high enough due to the relatively high oxygen partial pressure. Therefore, the ferromagnetic ordering is reduced due to the suppressed coupling of defects and dopants in this case. At room temperature some weakly coupled bound magnetic polarons are still paramagnetic. At low temperature, i.e., $5 \mathrm{~K}$, the paramagnetic polarons can change to ferromagnetic order [Fig. 11(b)]. In the case of the sample with the lowest defect concentration (the $\mathrm{PO}_{2}=10^{-3}$ sample), the isolated spins will not change to ferromagnetic ordering even at a temperature as low as $5 \mathrm{~K}$ [Fig. 11(c)]. Hence, there is only a small fraction of ferromagnetic phase at low temperature.

\section{CONCLUSION}

In this paper, we fabricated Co-doped $\mathrm{ZnO}$ thin films on quartz substrates with different oxygen partial pressures using a laser molecular beam epitaxy technique. XRD results confirm wurtzite structure and the growth orientation along the $c$ axis. TEM/EDS mapping indicate the uniform distribution of $\mathrm{Co}$ atoms in the $\mathrm{ZnO}$ films. No Co clusters are observed. XRD analysis and UV absorbance show the effective incorporation 
of Co ions on $\mathrm{Zn}$ sites and confirm the structural uniformity of the samples. Magnetization measurement reveals that Codoped $\mathrm{ZnO}$ films possess ferromagnetic ordering and the saturation magnetization increases with decreasing oxygen partial pressure. $\mu$ SR measurements also show that Co-doped $\mathrm{ZnO}$ films deposited at $10^{-7}$ torr have strong ferromagnetic ordering up to room temperature with a volume fraction of the ferromagnetic phase of $70 \%$, whereas the samples deposited under $\mathrm{PO}_{2}=10^{-3}$ and $10^{-5}$ torr mainly show paramagnetic behavior at room temperature. Resistivity versus temperature curves and $\mu \mathrm{SR}$ measurements both indicate that bound magnetic polarons are the origin of magnetic moments.
We propose that the interaction between magnetic polarons and oxygen vacancies leads to long range ferromagnetic coupling.

\section{ACKNOWLEDGMENTS}

J.Y. acknowledges the support of the Australia Research Council discovery Project Grants DP140103041 and Future Fellowship FT160100205. The $\mu$ SR measurements were performed at the Swiss Muon Source $\mathrm{S} \mu \mathrm{S}$, Paul Scherrer Institute, Villigen, Switzerland. J.Y. acknowledges the support of LMBE from ANFF, UNSW.
[1] S. Datta and B. Das, Appl. Phys. Lett. 56, 665 (1990).

[2] D. D. Awschalom and E. Flatté, Nat. Phys. 3, 153 (2007).

[3] R. Fiederling, M. Keim, G. Reuscher, W. Ossau, G. Schmidt, A. Waag, and L. W. Molenkamp, Nature (London) 402, 787 (1999).

[4] T. Dietl, H. Ohno, F. Matsukura, J. Cibert, and D. Ferrand, Science 287, 1019 (2000).

[5] H. Ohno, Science 281, 951 (1998).

[6] H. Ohno, J. Magn. Magn. Mater. 200, 110 (1999).

[7] T. Dietl, H. Ohno, and F. Matsukura, Phys. Rev. B 63, 195205 (2001).

[8] L. Chen, X. Yang, F. Yang, J. H. Zhao, J. Misuraca, P. Xiong, and S. von Molnár, Nano Lett. 11, 2584 (2011).

[9] R. O. Kuzian, A. M. Dare, P. Sati, and R. Hayn, Phys. Rev. B 74, 155201 (2006).

[10] Y. Matsumoto, M. Murakami, T. Shono, T. Hasegawa, T. Fukumura, M. Kawasaki, P. Ahmet, T. Chikyow, S. Koshihara, and H. Koinuma, Science 291, 854 (2001).

[11] K. Ueda, H. Tabata, and T. Kawai, Appl. Phys. Lett. 79, 988 (2001).

[12] N. H. Hong, J. Magn. Magn. Mater. 303, 338 (2006).

[13] T. S. Herng, D.-C. Qi, T. Berlijn, J. B. Yi, K. S. Yang, Y. Dai, Y. P. Feng, I. Santoso, C. Sanchez-Hanke, X. Y. Gao, A. T. S. Wee, W. Ku, J. Ding, and A. Rusydi, Phys. Rev. Lett. 105, 207201 (2010).

[14] X. Luo, W. T. Lee, G. Z. Xing, N. N. Bao, A. Yonis, D. W. Chu, J. Lee, J. Ding, S. Li, and J. B. Yi, Nanoscale Res. Lett. 9, 1 (2014).

[15] W. Prellier, A. Fouchet, and B. Mercey, J. Phys: Condens. Matter 15, R1583 (2003).

[16] M. S. Park and B. I. Min, Phys. Rev. B 68, 224436 (2003).

[17] S. Duhalde, M. F. Vignolo, F. Golmar, C. Chiliotte, C. E. Rodríguez Torres, L. A. Errico, A. F. Cabrera, M. Rentería, F. H. Sánchez, and M. Weissmann, Phys. Rev. B 72, 161313(R) (2005).

[18] J. Philip, A. Punnoose, B. I. Kim, K. M. Reddy, S. Layne, J. O. Holmes, B. Satpati, P. R. LeClair, T. S. Santos, and J. S. Moodera, Nat. Mater. 5, 298 (2006).

[19] Y. Tian, Y. F. Li, M. He, I. A. Putra, H. Y. Peng, B. Yao, S. A. Cheong, and T. Wu, Appl. Phys. Lett. 98, 162503 (2011).

[20] J. M. D. Coey, M. Venkatesan, and C. B. Fitzgerald, Nat. Mater. 4, 173 (2005).

[21] J. H. Park, M. G. Kim, H. M. Jang, S. Ryu, and Y. M. Kim, Appl. Phys. Lett. 84, 1338 (2004).
[22] S. Zhou, K. Potzger, Q. Xu, K. Kuepper, G. Talut, D. Markó, A. Mucklich, M. Helm, J. Fassbender, E. Arenholz, and H. Schmidt, Phys. Rev. B 80, 094409 (2009).

[23] D. Huang, Y. J. Zhao, D. H. Chen, and Y. Z. Shao, Appl. Phys. Lett. 92, 182509 (2008).

[24] K. Potzger, K. Kuepper, Q. Xu, S. Zhou, H. Schmidt, M. Helm, and J. Fassbender, J. Appl. Phys. 104, 023510 (2008).

[25] D. Iusan, M. Kabir, O. Granas, O. Eriksson, and B. Sanyal, Phys. Rev. B 79, 125202 (2009).

[26] T. Tietze, P. Audehm, Y.-C. Chen, G. Schátz, B. B. Straumal, S. G. Protasova, A. A. Mazilkin, T. Prokscha, H. Luetkens, Z. Salman, A. Suter, B. Baretzky, K. Fink, W. Wenzel, D. Danilov, and E. Goering, Sci. Rep. 5, 8871 (2014).

[27] J. Y. Kim, J. H. Park, B. G. Park, H. J. Noh, S. J. Oh, J. S. Yang, D. H. Kim, S. D. Bu, T. W. Noh, H. J. Lin, H. H. Hsieh, and C. T. Chen, Phys. Rev. Lett. 90, 017401 (2003).

[28] S. Yin, M. X. Xu, L. Yang, J. F. Liu, H. Rosner, H. Hahn, H. Gleiter, D. Schild, S. Doyle, T. Liu, T. D. Hu, E. TakayamaMuromachi, and J. Z. Jiang, Phys. Rev. B 73, 224408 (2006).

[29] A. Ney, K. Ollefs, S. Ye, T. Kammermeier, V. Ney, T. C. Kaspar, S. A. Chambers, F. Wilhelm, and A. Rogalev, Phys. Rev. Lett. 100, 157201 (2008)

[30] N. H. Hong, J. Sakai, N. Poirot, and V. Brizé, Phys. Rev. B 73, 132404 (2006).

[31] E. Z. Liu, Y. He, and J. Z. Jiang, Appl. Phys. Lett. 93, 132506 (2008).

[32] L. Shen, S. W. Yang, S. C. Xiang, T. Liu, B. C. Zhao, M. F. Ng, J. Goettlicher, J. B. Yi, S. Li, L. Wang, and Y. P. Feng, J. Am. Chem. Soc. 134, 17286 (2012).

[33] Y. W. Ma, Y. H. Lu, J. B. Yi, Y. P. Feng, T. S. Herng, X. Liu, D. Q. Gao, D. S. Xue, J. M. Xue, J. Y. Ouyang, and J. Ding, Nat. Commun. 3, 727 (2012).

[34] Y. R. Wang, X. Luo, L. T. Tseng, Z. M. Ao, T. Li, G. Z. Xing, N. N. Bao, K. Suzukiis, J. Ding, and S. Li, Chem. Mater. 27, 1285 (2015).

[35] G. Z. Xing, Y. H. Lu Y. F. Tian, J. B. Yi, C. C. Lim, Y. F. Li, G. P. Li, D. D. Wang, B. Yao, J. Ding, Y. P. Feng, and T. Wu, AIP Adv. 1, 022152 (2011).

[36] M. Fonin, G. Mayer, E. Biegger, N. Janben, M. Beyer, T. Thomay, R. Bratschitsch, Y. S. Dedkov, and U. Rudiger, J. Phys.: Conf. Ser. 100, 042034 (2008)

[37] N. Khare, M. J. Kappers, M. Wei, M. G. Blamire, and J. L. MacManus-Driscoll, Adv. Mater. 18, 1449 (2006). 
[38] J. B. Yi, C. C. Lim, G. Z. Xing, H. M. Fan, L. H. Van, S. L. Huang, K. S. Yang, X. L. Huang, X. B. Qin, B. Y. Wang, T. Wu, L. Wang, H. T. Zhang, X. Y. Gao, T. Liu, A. T. S. Wee, Y. P. Feng, and J. Ding, Phys. Rev. Lett. 104, 137201 (2010).

[39] Y. W. Ma, J. Ding, J. B. Yi, H. T. Zhang, and C. M. Ng, J. Appl. Phys. 105, 07C503 (2009).

[40] L. Li, Y. Guo, X. Y. Cui, R. Zheng, K. Ohtani, C. Kong, A. V. Ceguerra, M. P. Moody, J. D. Ye, H. H. Tan, C. Jagadish, H. Liu, C. Stampfl, H. Ohno, S. P. Ringer, and F. Matsukura, Phys. Rev. B 85, 174430 (2012).

[41] Z. L. Lu, H. S. Hsu, Y. H. Tzeng, F. M. Zhang, Y. W. Du, and J. C. A. Huang, Appl. Phys. Lett. 95, 102501 (2009).

[42] B. Pal and P. K. Giri, J. Nanosci. Nanotechnol. 11, 9167 (2011).

[43] A. Yaouanc and P. Dalmasde Réotier, Muon Spin Rotation, Relaxation, and Resonance: Applications to Condensed Matter (Oxford University Press, Oxford, UK, 2011).

[44] E. Morenzoni, T. Prokscha, A. Suter, H. Luetkens, and R. Khasanov, J. Phys.: Condens. Matter, 16, S4583 (2004).

[45] S. R. Dunsiger, J. P. Carlo, T. Goko, G. Nieuwenhuys, T. Prokscha, A. Suter, E. Morenzoni, D. Chiba, Y. Nishitani, T. Tanikawa, F. Matsukura, H. Ohno, J. Ohe, S. Maekawa, and Y. J. Uemura, Nat. Mater. 9, 299 (2010).

[46] A. V. Boris, Y. Matiks, E. Benckiser,A. Frano, P. Popovich, V. Hinkov, P. Wochner, M. Castro-Colin, E. Detemple, V. K. Malik, C. Bernhard, T. Prokscha, A. Suter, Z. Salman, E. Morenzoni, G. Cristiani, H. U. Habermeier, and B. Keimer, Science 332, 937 (2011).

[47] E. Morenzoni, B. M. Wojek, A. Suter, T. Prokscha, G. Logvenov, and I. Božović, Nat. Commun. 2, 272 (2011).

[48] H. Saadaoui, Z. Salman, H. Luetkens, T. Prokscha, A. Suter, W. A. MacFarlane, Y. Jiang, K. Jin, R. L. Greene, E. Morenzoni, and R. F. Kiefl, Nat. Commun. 6, 6041 (2015).

[49] A. Suter, E. Morenzoni, T. Prokscha, B. M. Wojek, H. Luetkens, G. Nieuwenhuys, A. Gozar, G. Logvenov, and I. Božović, Phys. Rev. Lett. 106, 237003 (2011).

[50] T. Prokscha, E. Morenzoni, K. Deiters, F. Foroughi, D. George, R. Kobler, A. Suter, and V. Vranković, Nucl. Instr. Meth. A 595, 317 (2008).
[51] T. Zywietz, J. Neugebauer, and M. Scheffler, Appl. Phys. Lett. 73, 487 (1998).

[52] C. Liu, S. H. Chang, T. W. Noh, M. Abouzaid, P. Ruterana, H. H. Lee, D. W. Kim, and J. S. Chung, Appl. Phys. Lett. 90, 011906 (2007).

[53] J. Ohta, H. Fujioka, M. Oshima, K. Fujiwara, and A. Ishii, Appl. Phys. Lett. 83, 3075 (2003).

[54] J. W. Shin, J. Y. Lee, Y. S. No, T. W. Kim, and W. K. Choi, J. Mater. Res. 24, 2006 (2009).

[55] L. P. The, V. Roger, C. David, X. N. Nguyen, and V. V. Ursaki, Nanotechnology 19, 475702 (2008).

[56] X. Qiu, L. Li, and G. Li, Appl. Phys. Lett. 88, 114103 (2006).

[57] M. Tay, Y. H. Wu, G. C. Hang, Y. B. Cheb, X. Q. Pan, S. J. Wang, P. Yang, and Y. P. Feng, J. Mater. Sci.: Mater. Electron. 20, 60 (2008).

[58] G. Singh, S. B. Shrivastava, and V. Ganesan, J. Chem. Eng. Mater. Sci. 4, 1 (2013).

[59] M. Bouloudenine, N. Viart, S. Colis, and A. Dinia, Chem. Phys. Lett. 397, 73 (2004).

[60] A. C. Tuan, J. D. Bryan, A. B. Pakhomov, V. Shutthanandan, S. Thevuthasan, D. E. McCready, D. Gaspar, M. H. Engelhard, J. W. Rogers, Jr., K. Krishnan, D. R. Gamelin, and S. A. Chambers, Phys. Rev. B 70, 054424 (2004).

[61] A. L. Efros and B. I. Shklovskii, J. Phys. C 8, L49 (1975).

[62] Y. F. Tian, S. S. Yan, Y. P. Zhang, H. Q. Song, G. Ji, G. L. Liu, Y. X. Chen, and L. M. Mei, J. Appl. Phys. 100, 103901 (2006).

[63] W. Eckstein, Computer Simulation of Ion-Solid Interactions (Springer, Berlin, Heidelberg, 1991).

[64] E. Morenzoni, H. Glückler, T. Prokscha, R. Khasanov, H. Luetkens, M. Birke, E. M. Forgan, Ch. Niedermayer, and M. Pleines, Nucl. Instr. Meth. B 192, 254 (2002).

[65] H. Saadaoui, X. Luo, Z. Salman, X. Y. Cui, N. N. Bao, P. Bao, R. K. Zheng, L. T. Tseng, Y. H. Du, T. Prokscha, A. Suter, T. Liu, Y. R. Wang, S. Li, J. Ding, S. P. Ringer, E. Morenzoni, and J. B. Yi, Phys. Rev. Lett. 117, 227202 (2016).

[66] A. Suter and B. M. Wojek, Phys. Proc. 30, 69 (2012).

[67] G. Z. Xing, J. B. Yi, J. G. Tao, T. Liu, L. M. Wong, Z. Zhang, G. P. Li, S. J. Wang, J. Ding, T. C. Sum, C. H. A. Huan, and T. Wu, Adv. Mater. 20, 3521 (2008). 